\title{
ESTADO DE SALUD ORAL EN EL ECUADOR
} \section{ORAL HEALTH STATUS IN ECUADOR}

\author{
Cabeza-Bernhardt Gerson ${ }^{1}$, González-Andrade Fernanda ${ }^{2}$, Paredes-Andrade Claudio $^{3}$ \\ ${ }^{1} \mathrm{Mg}$, Director del Post Grado de Ortodoncia Universidad San Francisco de Quito. Ecuador \\ ${ }^{2}$ Docente de la Universidad Católica de Cuenca. Ecuador \\ ${ }^{3}$ Odontólogo egresado de la Universidad Católica de Cuenca. Ecuador \\ *gersoncabezas@me.com
}

\begin{abstract}
Resumen
A lo largo de la historia del Ecuador, el sistema de salud pública se centraba en el control de las enfermedades, es decir, que giraban los tratamientos a la aparición de la enfermedad, sin contar con los recursos deficientes que tenía el estado, desfavoreciendo a la población más pobre. La patología más frecuente que se han presentado en el país ha sido la caries dental teniendo una relación con los determinantes sociodemográficos que influyen a cada individuo, sin embargo, en la actualidad el concepto del Ministerio de Salud Pública ha sido la prevención de la enfermedad y promoción de la salud, obteniendo así una mayor relación entre el paciente y el profesional odontológico.
\end{abstract}

Palabras clave: determinantes sociales,equidad, prevención, promoción, salud integral.

\begin{abstract}
Throughout the history of Ecuador, the health system was focused on the control of diseases, which is the treatment only over the appearance of the disease. Non-counting the limited resources the government has, disadvantaging the unfortunate poor population. The most frequent pathology presents in the country is dental caries. It has a relation with the socio demographic factors that influence each individual; however the present concept of public health system has been the prevention of the disease and promotion of health. Obtaining a greater relationship between the patient and the dental professional.
\end{abstract}

Key words: Social determinants, equity, prevention, promotion, integral health.

\section{ESTADO DE SALUD ORAL EN EL ECUADOR}

\subsection{ANTECEDENTES}

En las décadas anteriores en el Ecuador, el sistema nacional de salud estaba dirigido a los servicios que proporcionaba el Ministerio de Salud Pública, se centraban en el control de las enfermedades que se presentaban dentro de una comunidad, es decir, las acciones estaban centradas en la enfermedad, el curso que esta tenía, su incidencia, su frecuencia y distribución; datos que se obtenían de la atención de las patologías en las unidades hospitalarias consiguiendo de esta manera el control de padecimientos.

Uno de los limitantes era el acceso de la población a los servicios de salud debido a los costos económicos que debían ser cubiertos al momento de recibir atención, desfavoreciendo así a la población que se ubicaba en los quintiles más bajos de pobreza, faltando con ello a la declaración de Alma Ata donde se reconoce a la Atención Primaria de Salud como un derecho universal de todos. El Ecuador firmó y ratificó su participación considerando de esta manera la necesidad de una acción urgente por parte de todos los gobiernos.
Este también era el caso en el que se hallaba la salud bucal, debido a los altos costos de los centros médicos para poder generarla y ofertarla sus servicios. El Estado debe conservar la salud integral con la inversión de un alto presupuesto que debía ser otorgado al Ministerio de Salud Pública en su totalidad, debido a esta proporción del financiamiento se conseguía por parte de los pacientes que demandaban servicios odontológicos; a pesar de que los rubros que se cobraban en los servicios de salud públicos eran bajos comparados con los servicios de salud privados, se profundizó la inequidad en el acceso que tenía la población para obtener un estado de inmunidad adecuado, en especial para aquellos sectores de extrema pobreza, los cuales jamás pudieron adquirir un estado integral en la salud en sus comunidades.

La obligatoriedad de la asistencia al Plan Nacional de salud rural establecida para todos los estudiantes egresados de las facultades de Medicina, Enfermería, Obstetricia, Odontología (lo cual generó un acercamiento de los profesionales de salud a las comunidades de bajos recursos dejando como resultado un proceso de concienciación de las verdaderas necesidades que existen en poblaciones de extrema pobreza). El 
Plan de Desarrollo Integral de Odontología busca el progreso científico en este campo; la creación de la División Nacional de Odontología cuyo principal propósito es desarrollar programas de higiene y educación en medidas preventivas en comunidades rurales en conjunto con el desarrollo del Plan Nacional de Salud Bucal, son hechos trascendentales que establecieron normas para los servicios de salud y consolidaron al área de Salud Bucal como eje transversal para poder alcanzar un estado de salud integral de la población ecuatoriana. $^{1-3}$

Se puede decir también que desde la creación de la Carta de Ottawa principal documento basado en la prevención de las enfermedades, la manera que los servicios de salud ambulatorios y hospitalarios entienden que los procesos de salud - enfermedad han cambiado no solo en el Ecuador sino también en el resto del mundo. La nueva visión de cómo se debe enfrentar una patología desde antes que ésta se presente produjo un descenso en la cantidad de pacientes que adquirieron cronicidad de una enfermedad evitable. La prevención primaria (generada antes de que aparezca la enfermedad dada para reducir la incidencia), secundaria (generada para disminuir rápidamente los síntomas generados en una patología ya adquirida y poder erradicarla) y terciaria (generada para lograr la adecuada reinserción de los pacientes a la sociedad después de haber sufrido el curso de una enfermedad) son las principales armas que los profesionales de la salud usan hoy en día. ${ }^{4}$

\subsection{PRINCIPALES PATOLOGÍAS}

Al hablar de las principales patologías que aquejan a la población en primer lugar se ubica la caries dental, la cual ha sido una patología conocida a lo largo de la historia de la humanidad siendo en un principio no diferenciada como un problema grave debido al desconocimiento de las causa, factores de riesgo y curso de esta patología, es así como la caries adquirió importancia como problema de salud pública debido al alto consumo de azúcar refinada registrado en los países de primer mundo, ya que en dichos países se comenzó la industrialización de la caña para obtener el azúcar; esto a su vez originó en la población de estos países el aumento en la demanda de servicios odontológicos como resultado de que en esta época hubieron reportes en el que no existían un solo infante sin incidencia de caries, por lo que se puede decir que, la principal demanda dentro del cuidado estomatológico son las caries.

En el Ecuador se han desarrollado diferentes estudios acerca de cuales son las principales patologías que afectan a los individuos en cuanto a la salud bucal se refiere, los resultados arrojados en dichas investigaciones son similares a otros estudios efectuados en países de América Latina indican que las caries, la placa bacteriana, la gingivitis, las periodontopatías y la fluorosis dental son las pricipales patologías que afecta a su población. ${ }^{4}$
Siendo así el orden de las principales patologías que afecta a la población Latinoamericana

Debido a lo anteriormente mencionado se debió implementar tratamiento odontológico para cada caso, según la severidad del daño que éste haya ocasionado en las piezas dentales, por ejemplo, en la planificación de prevención primaria se requiere brindar información adecuada (científica y comprobable) acerca de la patología, su proceso y sus posibles consecuencias además de manejar como principal herramienta la profilaxis dental, la cual ayudará a prevenir posibles patologías mórbidas. En cuanto a la prevención secundaria se refiere a los procesos operatorios dentales, endodoncias y exodoncias. Para finalizar en la prevención terciaria deberemos ayudar al paciente en el proceso de rehabilitación física y emocional brindando tratamientos en prótesis, lo cual ayudará a que la reinserción social que sea más fácil y tenga mejores resultados.

\subsection{DETERMIANTES SOCIALES}

Los determinantes de la salud ayudarán a potencializar y generar protección contra el daño que pueda generar una patología, estos se pueden encontrar bajo un contexto socioeconómico, cultural y político. En cuanto al aspecto político encontramos como principal actor el gobierno, el cual mediante sus diferentes entidades generará políticas macroeconómicas, sociales, públicas, entre otros; los cuales ayudarán a que la población tenga acceso a los servicios médicos que requieran entre ellos, y uno de los más necesitados es el servicio odontológico. Los determinantes sociodemográficos como la posición socioeconómica de los individuos se diferencian por las clases sociales, el género, la etnia, así como la educación, ocupación y los ingresos que ésta genera, permitiendo medir cual es el número de pacientes que solicitan la atención y que clase de atención requieren. Existen también los denominados determinantes culturales intermedios de la salud, entre los que podemos encontrar los factores conductuales que se refieren a los diferentes hábitos y costumbres que tienen las personas y que pueden potencializar un daño o un estado de salud; los factores biológicos de las personas se refieren a su carga genética, lo cual marcará una diferencia significativa para la adquisición de patologías debido a la suceptibilidad biológica de cada persona. Para finalizar los determinantes ambientales, en los cuales la interacción de los individuos con el medio, marcan la generación de condiciones de protección o riesgo para la salud.

Se debe tomar en cuenta que la salud bucal se ha constituido como un factor de suma importancia en cuanto al ámbito de bienestar bio- psico- social de los individuos, ya que este repercute en cuanto a la calidad de vida de las personas y su estado de salud integral, es por eso que al hacer énfasis en los determinantes de salud para tener un claro panorama de cuales serían las deficiencias dentro del sistema nacional de salud y principalmente en cuanto a la atención estomatológica se refiere, debido a que si en 
una persona se encuentra deteriorado su ámbito biológico (nos referimos exclusivamente al ámbito estomatológico) no podrá decirse que posee un estado de salud integral, además se ha demostrado que produce una decaída en el aspecto personal, los individuos afectados sufren de baja autoestima y esto en la mayoría de los casos se ve repercutido debido a la recepción sensorial que tienen los demás individuos, esto en el aspecto social. En virtud de esto se podrá realizar planes, programas, proyectos de Salud Oral en los cuales se pueda promover el fortalecimiento de estos determinantes de la salud y conocer los factores de riesgo, precipitantes para poder contrarrestarlos adecuadamente y luego de manera inmediata hacer énfasis en los factores protectores con los que cada individuo y comunidad cuenta para reforzar así la obtención de un estado de salud integral, también se debe mediante la educación de la salud bucal modificar actitudes, por ejemplo, asistir a revisiones odontológicas periódicas para el control y evaluación de la salud oral de los individuos, hábitos especialmente en cuanto al correcto uso del cepillado dental se refiere como las veces que cada día debe ser efectuado, mejorar la nutrición en los miembros de la comunidad reduciendo así las dietas cardiogénica y de esta manera reducir la incidencia de la caries.

\subsection{ACTUALIDAD.}

En la actualidad el gobierno nacional basado en diferentes normativas legales principalmente en la Constitución del Ecuador, la cual plantea que la salud es un derecho que debe garantizar el estado, además de definir los grupos de atención prioritaria y establecer que los servicios de salud deben ser de carácter universal y gratuito, también promueve la participación de la comunidad en todos los ámbitos. El estado dispuesto hacer de la salud un bien público, basado en el análisis de los estudios epidemiológicos realizados acerca de las principales patologías que afectan a los individuos dentro del territorio nacional, se ha procurado la realización de un plan nacional de salud bucal con enfoque en la promoción y prevención, procurando la eliminación de las disparidades sanitarias. El plan nacional de salud bucal se basa en los siguientes principios: Integridad en la atención, intersectorialidad, accesibilidad, equidad, derecho a la salud bucal, continuidad en la atención, calidad, simplificación de técnicas y calidez; Esto garantizará el acceso de la población ecuatoriana a la salud bucal, la cual solo mediante educación en todos los aspectos que esta requiere logrará modificar actitudes y hábitos, en cuanto a tipo de alimentación y comportamientos, siempre teniendo en cuenta los factores como género, etnia, edad, clase social; entre otros; los cuales mediante los servicios de vigilancia epidemiológica nos brindarán resultados positivos.

Entre las actividades que se procurará tener la normatización, la cual nos permitirá elaborar un plan estratégico con los delegados de los diferentes sectores del medio odontológico para poder en conjunto elaborar manuales de biosegu- ridad, lineamientos de bioética aplicables a la odontología, elaborar también protocolos adecuados de atención en los diferentes niveles y ciclos de vida. El Sistema de Información nos brindará el análisis de los datos estadísticos para poder de una manera bidireccional conocer las principales patologías estomatológicas que están afectando a la comunidad mediante la revisión de historias clínicas, la emisión de informes diarios y mensuales.

El papel de los recursos humanos en cuanto a los profesionales de la salud bucal juega un rol fundamental en el proceso de la generación nacional de una salud integral, debido a que estos no solo deben establecerse en cuanto al servicio odontológico, es decir, no solo en la que se genere en los consultorios de los centros médicos, sino que también se debe proveer por parte de las autoridades capacitación constante en dos aspectos principales:

a) Planificación y estrategias de prevención y acercamiento con la comunidad es de alta importancia.

b) Capacitación en los diferentes avances tecnológicos y técnicas nuevas generará un mejor desempeño en los profesionales y mayor confianza en la comunidad.

La formación de pregrado de Odontología deberá establecerse con énfasis en promoción y prevención de la Salud bucal y no solo ahondar en la formación de manejo de morbilidad, la formación de pregrado deberá dar un giro completo asegurando con ello que los profesionales priorizaran y enfatizaran brindar una atención preventiva y que además de ello estarán en la entera capacidad de diseñar estrategias que reduzcan la aparición de enfermedades bucales identificadas en la comunidad. La vigilancia epidemiológica y evaluación de programas preventivos de la atención odontológica y de la identificación de grupos de riesgo no debe ser regido solamente por los resultados de prestación de servicios, sino que debe hacer énfasis en como el proceso fue ejecutado y las determinantes de la calidad de la atención como: aspectos técnicos científicos, la percepción del usuario, entre otros y de esta manera tener resultados objetivos con los cuales medir los avances que se dan, en cuanto a la atención y el desarrollo de un mejor estado de salud; por lo cual no debemos olvidar que la vigilancia epidemiológica también debe dirigirse al reconocimiento de patrones cambiantes en los aspectos sociodemográficos de una comunidad y los cursos de las enfermedades.

\section{FUNCIONES, ACTIVIDADES PARA EL ODONTÓ- LOGO DEL SERVICIO INTEGRAL DE SALUD.}

Los profesionales del área de Odontología dentro del Plan Nacional de Salud Bucal deben tener como enfoque principal la promoción (creación de políticas y protocolos adecuados para reducir los índices de incidencia de las enfermedades estomatológicas) y la prevención (primaria, secundaria y terciaria) de la Salud Oral en los individuos de 
las comunidades, la generación de programas de promoción de la salud bucal debe encaminarse a desarrollar hábitos saludables (correcta limpieza de dientes y encías, adecuada técnica de cepillado, control de placa bacteriana por parte de los individuos y los profesionales en una revisión periódica del estado de salud bucal, uso de hilo dental como accionar complementario y obligatorio), educación para el conocimiento de la importancia de la lactancia materna y la seguridad alimentaria en los aspectos nutritivos (dando como resultado un mejoramiento en el sistema inmunitario y un reforzamiento en el aspecto biológico) y de dietas bajas en agentes cariogénicos lo cual reducirá el índice, prevalencia e incidencia de casos. El trabajo de los profesionales de la salud oral también tendrá la obligación de dirigirse en prevención primaria a contrarrestar las principales patologías antes de su aparecimiento en revisiones periódicas en los consultorios odontológicos de los servicios públicos de salud, en lo cual se necesita hacer una detección temprana de la placa bacteriana, enseñar el correcto procedimiento del cepillado dental y de ser necesario la aplicación de sellantes, esto basados en un diagnóstico temprano y por lo tanto sin permitir la cronicidad de las enfermedades estomatológicas, por lo cual es necesario la profilaxis bucal por parte de los profesionales. En cuanto a la educación en higiene bucal, los individuos deben regir a cada etapa del ciclo de la vida de las personas ya que en cada una de estas fases tanto los factores de riesgo como las necesidades, actitudes y comportamientos de los sujetos varían; es así como en la primera etapa, los bebés de 0 a 2 años se ven marcada en el inicio de la dentición temporal, es por eso que el trabajo principal es con los padres del niño, enfocando en los cuidados que se deben realizar en casa. En los niños preescolares, es decir de 3 a 5 años de edad, el cepillado dental es de absoluta responsabilidad de los padres, debido a que los menores se encuentran en la etapa de adquisición de hábitos, los padres son los encargados de enseñar correctamente la forma en la cual se deben cepillar los dientes, y de ser necesario acudir a los centros pediátrico odontológicos para informarse acerca de técnicas lúdicas con las que podrá generar motivación y una mejor adaptación al estilo de vida, además de crear un vínculo temprano entre los profesionales odontólogos y los individuos con lo que se podrá generar desde edades tempranas empatía en los niños hacia los procesos de salud oral. De 6 a 14 años, ya debe existir una regularidad en la interacción del cuidado en el hogar, haciendo responsable al niño de su cepillado y enseñando el correcto proceso de éste y la atención en el consultorio odontológico va dirigida al cuidado de la salud oral por medio de la profilaxis, asesoría técnica, educación acerca del uso de hilo dental. En los adolescentes y adultos se debe desarrollar en casa, el uso de pasta dental para implementar un correcto cepillado. El profesional deberá proporcionar un control semestral del estado de salud bucal de las personas, además de brindar los informes individuales a los pacientes deberán enviar informes a los centros de acopio de información de los servicios de vigilancia epidemiológica para poder realizar el análisis oportuno de los datos adquiridos y poder emplear en futuros programas y proyectos dirigidos a la prevención de patologías. En el 2012, en uno de los boletines emitidos por la Organización Mundial de la Salud refiere que, alrededor del $30 \%$ de la población mundial con edades comprendidas entre los 65 y los 74 años no tiene dientes naturales. Entendiendo con ello que, la población presenta una condición disminuida en la estética y función del sistema estomatognatico. Además de ésto, la OMS considera a la caries dental como una enfermedad prevenible de la cavidad bucal, resaltando con ello la gran importancia de poner en marcha políticas y estrategias públicas que garanticen la ausencia de la enfermedad.

\section{CONCLUSIONES}

- Debido a la alta prevalencia, incidencia y la rápida distribución existente en las diferentes patologías estomatológicas mencionadas a través de este estudio el gobierno nacional a procurado la creación de políticas adecuadas como El Plan Nacional del Buen Vivir, El plan Nacional de Salud Bucal, El Plan Nacional de Salud Rural, y por sobre todas la Constitución Nacional de la República del Ecuador en las que se desea alcanzar la equidad de la población en cuanto a obtener la salud integral y el uso de los servicios de salud, que el MSP ha provisto en los diferentes sectores del territorio nacional para la rápida atención de los requerimientos generados en las comunidades, no solo urbanas sino que se ha procurado énfasis en aquellas alejadas de las grandes ciudades.

- La sola creación de políticas estatales no ha sido suficiente para lograr alcanzar el estado de salud integral, en especial en el área estomatológica debido a que existen factores económicos relacionados con los profesionales odontólogos y con la población que no han podido ser subsanados. En cuanto al factor económico, el Estado ha generado un mayor presupuesto para que de esta manera lograr reforzar el aspecto estructural de los hospitales, centros y subcentros de salud, además a provisto de insumos requeridos basados en los estudios de las necesidades de la población para poder cubrir estas y lograr la satisfacción de los ciudadanos que asisten a los servicios hospitalarios públicos, en última y no menos importante instancia el Gobierno a procurado a más de el mejoramiento de las instalaciones y de la adecuada adquisición de insumos, también implementar equipos tecnológicos de punta que ayudan en el mejor desempeño de los profesionales en el área de Estomatología.

- La capacitación constante de los servidores públicos del servicio estomatológico también ha sido una constante en la actual administración gubernamental, ya que de esta manera se ha podido mejorar el servicio a la comunidad, para tener una mejor calidad en la atención que hoy por hoy se maneja bajo los estándares de calidad y calidez. 
- El trabajo junto a la comunidad y el profesional Odontólogo deberá estar relacionado especialmente a la prevención primaria de los individuos miembros de la comunidad (pacientes), es así que los profesionales del área estomatológica de los servicios de salud pública adquieran responsabilidades puntuales en el proceso de adquirir salud bucal como parte esencial de la salud integral.

\section{PROPUESTA}

Los resultados de los estudios epidemiológicos de salud oral en Ecuador coinciden con los resultados obtenidos en varios países y concuerda también el grupo de patologías más comunes presentadas en la cavidad oral, por tanto, el lineamiento del estado está encajado en el lineamiento del Organización Mundial de la Salud, sin que esto signifique que se están cumpliendo a cabalidad las políticas y propuestas. El ministerio de salud se encuentra con una gran responsabilidad, los pocos estudios epidemiológicos nos muestran que en el país todavía existe mucho trabajo por hacer, pero la forma en que esta constituida la institución no le permite tener una respuesta rápida y ejecutiva para poder afrontar los problemas. La falta de cultura en los cuidados de salud oral son una de las principales causas para el deterioro de la salud en los ecuatorianos, esta debe ser afrontada por medio de leyes como:

- Obligación de los padres de llevar a sus hijos a chequeos odontológicos desde edades muy tempranas debiendo reportarlo en la ficha de vacunas que por ley deben tener todos los niños.

- Control por medio de ficha médica del ministerio de salud sobre la ingesta de suplementos vitamínicos durante el embarazo.

- Tener un programa de promoción de la salud oral completamente definido.

- Crear una fuente de ingresos independiente de otras políticas de estado que sea permanente y suficiente para realizar la promoción, la prevención, sobre todo a la atención primaria en salud oral

- Concientizar sobre la educación oral como una asignatura permanente en la formación educativa de preescolar, escolar, secundaria y de tercer nivel.

Conflicto de intereses y financiamiento Los autores declaran no tener conflicto de intereses, haber cumplido con los requisitos de autoría y haber autofinanciado este artículo.

\section{Referencias}

1 Pinto G. Ministerio de Salud Pública del Ecuado, Ed. Plan nacional de salud bucal . 1 ed., Vol. 1. Quito, Pichincha, Ecuador; 2009.

2 Ministerio de Salud Pública del Ecuador. Manual del modelo de atención integral de salud. 1ed., Vol.2., Quito, Pichincha, Ecuador; 2013: 1-211.
3 Ministerio de Salud Pública del Ecuador, Organización Panamericana de la Salud. Estudio Epidemiológico de salud bucal en escolares fiscales menores de 15 Años en el Ecuador. Quito, Ecuador. 1995-1996: 1-44.

4 Organización Mundial de la Salud. Carta de Ottawa. OMS, Ottawa. 1992 Ley de maternidad gratuita y atención a la infancia - Ecuador (Codificación No. 2006-006)

5 The Pan American Health Organization Promoting Health in the Americas Declaracion de alma-ata Conferencia Internacional sobre Atención Primaria de Salud, Alma-Ata, URSS, 6-12 de septiembre de 1978-2007. Disponible en

Recibido: 18 de Mayo de 2016

Aceptado: 23 de Mayo de 2016 
